\title{
Image Recognition Technology with Its Application in Defect Detection and Diagnosis Analysis of Substation Equipment
}

\author{
Long Luo (D), ${ }^{1}$ Rukuo Ma, ${ }^{1}$ Yuan Li, ${ }^{1}$ Fangnan Yang, ${ }^{2}$ and Zhanfei Qiu ${ }^{1}$ \\ ${ }^{1}$ State Grid Qinghai Maintenance Company, Xining 810000, China \\ ${ }^{2}$ State Grid Qinghai Electric Power Company, Xining 810000, China \\ Correspondence should be addressed to Long Luo; lluo.sgqh@yahoo.com
}

Received 27 August 2021; Accepted 23 September 2021; Published 25 November 2021

Academic Editor: Bai Yuan Ding

Copyright (c) 2021 Long Luo et al. This is an open access article distributed under the Creative Commons Attribution License, which permits unrestricted use, distribution, and reproduction in any medium, provided the original work is properly cited.

Detection of substation equipment can promptly and effectively discover equipment overheating defects and prevent equipment failures. Traditional manual diagnosis methods are difficult to deal with the massive infrared images generated by the autonomous inspection of substation robots and drones. At present, most of the infrared image defect recognition is based on traditional machine learning algorithms, with low recognition accuracy and poor generalization capability. Therefore, this paper develops a method for identifying infrared defects of substation equipment based on the improvement of traditional ones. First, based on the Faster RCNN, target detection is performed on 6 types of substation equipment including bushings, insulators, wires, voltage transformers, lightning rods, and circuit breakers to achieve precise positioning of the equipment. Afterwards, different classes are identified based on the sparse representation-based classification (SRC), so the actual label of the input sample can be obtained. Finally, based on the temperature threshold discriminant algorithm, defects are identified in the equipment area. The measured infrared images are used for experiments. The average detection accuracy achieved by the proposed method for the 6 types of equipment reaches $92.34 \%$. The recognition rate of different types of equipment is $98.57 \%$, and the defect recognition accuracy reaches $88.75 \%$. The experimental results show the effectiveness and accuracy of the proposed method.

\section{Introduction}

The power station is an important node in the power grid that is responsible for converting voltage and distributing electric energy. Its safety and reliability are directly related to the safety and stability of the power system [1-5]. According to the statistics, about half of the power equipment failures have abnormal temperature in the early stage. Infrared detection of power equipment has the advantages of nonpower failure, noncontact, high sensitivity, and mature technology [6-8]. It can detect equipment overheating defects in a timely and effective manner and prevent equipment failures. It has been widely used in the field of power equipment thermal fault diagnosis. In recent years, infrared thermal imagers have been equipped with inspection platforms such as substation inspection robots and drones to conduct intelligent inspections on substation equipment, reducing the workload of operation and maintenance personnel to collect infrared images of equipment [9-14]. However, a large number of inspections or online monitoring still require manual analysis, which is time-consuming and inefficient, and also difficult to cope with the massive infrared images generated by inspections. Therefore, the researchers have carried out a series of works on infrared defect recognition. These methods used different types of features or classification models to analyze and process images and then determined and recognized defects. Most of the existing research studies on infrared image defect recognition are based on traditional machine learning algorithms, which have disadvantages such as low accuracy, poor generalization ability, and rigid model. With the development of artificial intelligence technology, image detection algorithms based on deep learning have been widely used in the field of visible light object detection [13-16], but there are 
few applications in infrared image recognition and detection. So, it is urgent to carry out infrared image-based fault identification algorithms of substation equipment [17-19].

Based on the improvement of traditional image analysis technology, this paper proposes an infrared defect recognition method for substation equipment. First, based on the Faster RCNN algorithm [20-23], target detection is performed on 6 types of substation equipment, including bushings, insulators, wires, voltage transformers, lightning rods, and circuit breakers, to achieve precise positioning of the equipment. Next, for the detected image area of the substation equipment, fine classification is performed based on the sparse representation-based classification (SRC) [24-27]. And, the specific substation equipment label to which it belongs is determined based on the training samples. Finally, based on the threshold on the temperature, defects are identified in the equipment area to determine whether the equipment has defects and the relevant severity. Compared with the traditional image defect detection algorithms of substation equipment, this paper further introduces SRC to realize the accurate confirmation of the category of the input substation equipment. Such operation has important auxiliary significance for subsequent targeted defect detection and failure analysis. In the experiment, the proposed method is tested based on the collecting infrared images from actual measurement to verify its feasibility and accuracy.

\section{Equipment Detection Based on Faster RCNN}

This paper first builds an infrared image defect detection model for substation equipment based on Faster RCNN. The specific frame structure is shown in Figure 1. First, the Faster RCNN detection model is trained based on the infrared image training dataset of the substation equipment to generate a defect detection network. Then, the test infrared image is input into the defect detection network to verify the detection performance. As shown in Figure 1, the Faster RCNN detection network is composed of two parts: a region proposal network (RPN) and a region convolutional neural network (RCNN). The Faster RCNN model is an integrated network that can effectively realize object detection in input images. It mainly completes region proposal and $\mathrm{CNN}$ detection, which are completed by RPN and RCNN, respectively. RPN is a fully convolutional neural network that can effectively predict object boundaries and object scores at various positions in the input image. Object boundaries and object scores are important indicators for evaluating whether an area is proposed. The image input proposal given by RPN will be input into RCNN to realize the classification of the proposed area and calculate the score of each proposed area. The Faster RCNN model integrates the RPN network and RCNN into one network by sharing convolutional features and provides a joint training method. Compared with a single network structure, the Faster RCNN model deepens the network depth and improves the image detection performance. There have been many research results based on Faster RCNN's target detection and power equipment detection [20-23]. This paper mainly uses the direct application shown in Figure 1 and will not introduce it in detail here.

\section{Equipment Recognition and Defect Diagnosis}

3.1. SRC. The basic principle of SRC [24-27] is to use training samples to linearly fit the test samples of unknown categories and constrain the high sparsity of linear representation coefficients. On this basis, the reconstruction error of each training class for the test sample is calculated separately to determine the target label of the test sample. Assume that the training samples of the $M$-class targets construct a global dictionary $A=\left[A^{1}, A^{2}, \ldots, A^{M}\right] \in R^{d \times N}$, where $A^{i} \in R^{d \times N_{i}}(i=1,2, \ldots, M)$ are all the training samples of the $i$ th class. For the test sample $y$ with an unknown target label, the sparse representation process is as follows:

$$
\begin{aligned}
\hat{x} & =\arg \min \|x\|_{0}, \\
\text { s.t. } y & =A x .
\end{aligned}
$$

In equation (1), $x$ is the linear coefficient vector to be solved. Under the sparsity constraint, only a small number of elements in the coefficients are nonzero. With the optimal estimated $\hat{x}$ to be obtained, equation (3) is used to calculate the reconstruction error of each training class for the test sample, and then, the decision is made according to the minimum error criterion:

$$
\begin{aligned}
r(i) & =\left\|y-A \delta_{i}(\widehat{x})\right\|_{2}^{2}, \quad i=1,2, \ldots, M, \\
\operatorname{identity}(y) & =\underset{i}{\arg \min }(r(i)),
\end{aligned}
$$

where $\delta_{i}(\hat{x})$ represents the sparse representation coefficient vector corresponding to the $i$ th class and $r(i)(i=1,2, \ldots, M)$ is the reconstruction error of the $i$ th class.

It can be seen that the key problem in SRC is the solution of the sparse coefficients. With the accurate and reliable solutions, the target label of the test sample can be accurately obtained. Since the problem of minimizing the $\ell_{0}$-norm in equation (1) is an NP-hard problem, it is difficult to solve it directly. Researchers found that the $\ell_{1}$-norm minimization optimization and the $\ell_{0}$-norm minimization optimization are equivalent under certain conditions [24], so equation (1) can be transformed into

$$
\begin{aligned}
\widehat{x} & =\arg \min \|x\|_{1}, \\
\text { s.t. } y & =A x .
\end{aligned}
$$

The introduction of the $\ell_{1}$ norm can turn the original problem into a convex optimization problem which is easy to solve [5]. So, the linear programming method can be used to solve the sparse representation coefficients. In the actual process, due to the influence of noise and the unstable error between the training and the test samples, it is difficult to achieve a completely accurate reconstruction. Therefore, the sparse coefficient vector is solved as follows under the given reconstruction error limit: 


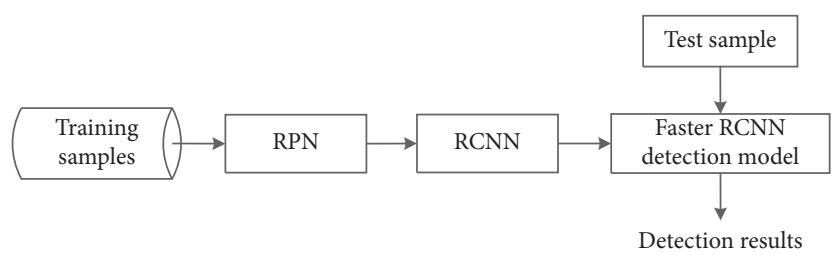

Figure 1: Detection process of substation equipment based on Faster RCNN.

$$
\begin{aligned}
& \widehat{x}=\arg \min \|x\|_{1}, \\
& \text { s.t. }\|A x-y\|_{2}<\varepsilon,
\end{aligned}
$$

where $\varepsilon$ is the allowable reconstruction error.

Based on the detection results of substation equipment by Faster RCNN, this paper uses SRC for precise identification for each region of the detected results. A global dictionary is constructed based on the training samples with known target labels. Then, the reconstruction error of the input sample in any class is calculated according to the abovementioned sparse representation and reconstruction process. According to the reconstruction errors, the substation equipment label is determined precisely.

3.2. Defect Diagnosis. Abnormal heating of substation equipment is an important basis for judging the defects. Frequent defects in substation equipment include defects, loose parts, loose wires, corrosion or oxidation of equipment contact surfaces, and excessive current-carrying. The equipment's own defects cause the equipment to fail to operate normally and heat up; then, the equipment must be replaced. Loose parts and loose wires are common failures of the capacitor interface accessories of the power transmission and transformation equipment. This type of failure is caused by the change in the area. Corrosion or oxidation of the equipment contact surface is a common fault in electrical contact parts such as disconnecting switches of power equipment. This type of fault increases current-carrying resistance and causes the fault point to heat up. When the current-carrying is too large, the equipment is under high load. After running for a certain period of time, abnormal heating will also occur.

Electricity can divide equipment thermal defects into current heating and voltage heating according to different heating properties. Current heating defects refer to the heating caused by the resistance of the current flowing through the conductive loop, which is mainly concentrated at the equipment connectors. And, the temperature rise is more obvious and easier to be found, accounting for about $90 \%$ of the total number of faults [12]. Voltage heating defect refers to the heating of the device caused by the action of the electric field when the operating voltage is applied to the insulating medium of the device. Only a small part of the heat of this type of fault can be conducted to the surface of the equipment shell, and the temperature rise that can be measured is very small, which is difficult to be detected. This paper proposes an infrared defect recognition method for current-induced defects. Current heating-type defects include joint heating, disconnection switch knife edge and switching hair heating, circuit breaker contact heating, transformer internal connection heating, and bushing string heating. The surface temperature discrimination method is the simplest and most effective method for judging current-heated defects. According to previous works, three temperature threshold values of $50^{\circ} \mathrm{C}, 80^{\circ} \mathrm{C}$, and $105^{\circ} \mathrm{C}$ are selected to identify defects in the equipment. Urgent defects represent defects that have a serious threat and need to be addressed immediately. A serious defect indicates a fault that may threaten to be dealt with as soon as possible. General deficiencies indicate that there is little threat to the safety of equipment operation that requires enhanced surveillance. On the basis of completing the detection and identification of substation equipment, the process of infrared image defect identification in this paper is shown in Figure 2.

\section{Experiments and Analysis}

4.1. Preparation. This paper selects infrared images of 6 types of substation equipment including bushings, insulators, wires, voltage transformers, lightning rods, and circuit breakers for defect detection. These images mainly come from the grid company reserves and collected data in actual scenes. The dataset includes a total of 800 defective images and 1900 normal images. In order to avoid additional deviations introduced in the data division process and affect the final result, the training set and the test set should be randomly selected from the original ones. Finally, this paper randomly chooses 1700 infrared images of substation equipment from the total sample set as the training set and 600 infrared images as the test set.

4.2. Results and Discussion. For Faster RCNN-based substation equipment detection, in the experiment, the model training learning rate is set to 0.001 , mini-batch size 256 , momentum 0.9, weight attenuation 0.0001, and total number of iterations 20000. The 1700 training samples are trained to obtain substation equipment detection models. The detection accuracy of 6 types of substation equipment achieved by the proposed method is shown in Table 1, and the average accuracy is $92.34 \%$. This result verifies the effectiveness of Faster RCNN for image detection of substation equipment.

The training samples are also used to train the SRC to obtain the corresponding classification model. According to the Faster RCNN results of the test samples, the concrete label of the substation equipment to which the test samples belong is confirmed. Table 2 shows the recognition rates of different types of substation equipment based on SRC, and the average recognition rate reaches $98.57 \%$. This result proves the effectiveness of SRC for the identification of substation equipment. 


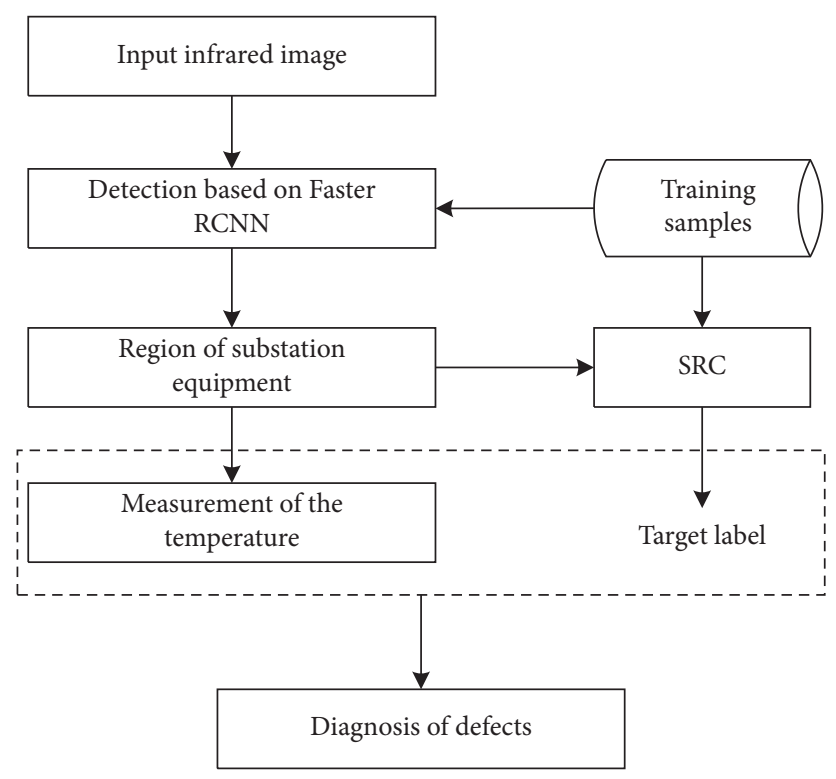

Figure 2: Procedure of defect diagnosis based on temperature.

TABLE 1: Analysis of detection performance based on Faster RCNN.

\begin{tabular}{lr}
\hline Equipment & Detection accuracy (\%) \\
\hline Bushing & 92.32 \\
Insulator & 93.46 \\
Wire & 91.23 \\
Voltage transformer & 93.56 \\
Lightning rod & 89.24 \\
Circuit breaker & 90.03 \\
\hline
\end{tabular}

TABLe 2: Analysis of recognition performance based on SRC.

\begin{tabular}{lr}
\hline Equipment & Recognition rate (\%) \\
\hline Bushing & 98.56 \\
Insulator & 98.92 \\
Wire & 97.31 \\
Voltage transformer & 97.85 \\
Lightning rod & 99.02 \\
Circuit breaker & 98.34 \\
\hline
\end{tabular}

On the basis of the abovementioned detection and identification process, the defect detection of substation equipment is carried out based on the idea of temperature thresholds. According to statistics, there are a total of 160 defective devices in the test set of 600 infrared images, and 142 devices are correctly judged as defective based on the proposed algorithm, with an accuracy rate of $88.75 \%$. Figure 3 shows examples of the defect detection results of the proposed method for some substation equipment. The region marked in the dashed box is the abnormal part where the temperature exceeds the set threshold, that is, the local area where defects may exist. The results are consistent with the actual situation. The experimental results show the effectiveness and accuracy of the method in this paper. In the specific defect processing process, because this article not only carried out the inspection of 


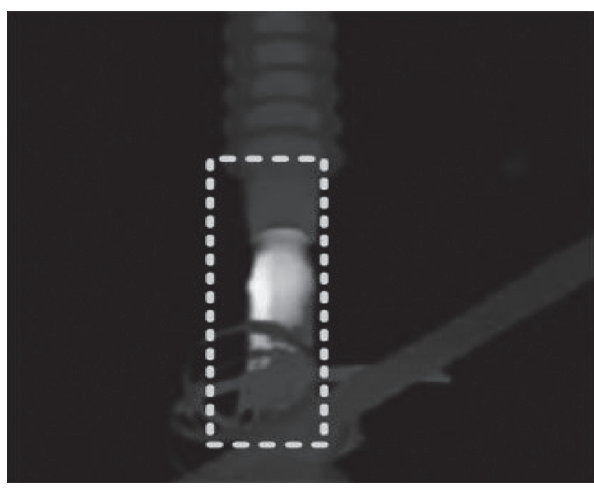

(a)

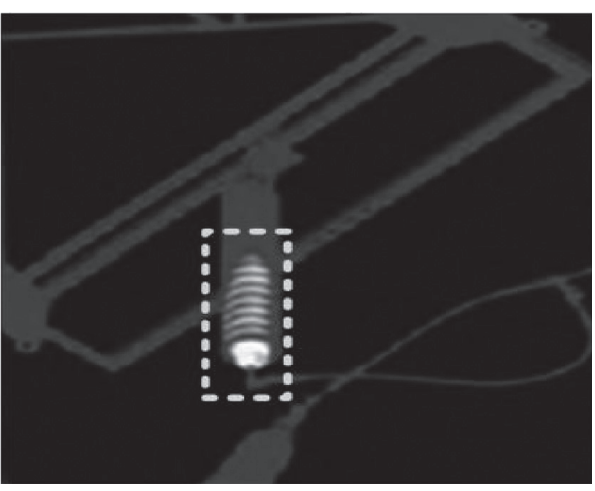

(b)

Figure 3: Examples of defect detection results.

the equipment but also confirmed its category, it can assist the staff to formulate more effective treatment measures.

\section{Conclusion}

This paper applies image recognition technology to the detection and diagnosis of defects in substation equipment. First, for an input infrared image of substation equipment, Faster RCNN is used to detect the key areas to obtain the substation equipment of interest. On this basis, relying on the training samples that have been classified, the detected area is confirmed based on the SRC, and the specific type of the substation equipment is determined. Finally, determine whether it is in a normal state according to the temperature of the device reflected in the current infrared image. Compared with traditional substation equipment defect analysis algorithms, this paper further confirms the category of input equipment by introducing SRC, so the analysis results are more informative, which is conducive to the development of targeted diagnosis and repair. Experiments are carried out on the infrared image set of actual measurement substation equipment, and the results show the effectiveness of the proposed method for defect detection and identification of substation equipment.

\section{Data Availability}

The data used in this study are available from the authors upon request.

\section{Conflicts of Interest}

The authors declare that there are no conflicts of interest.

\section{References}

[1] G. C. Stone, "Partial discharge diagnostics and electrical equipment insulation condition assessment," IEEE Transactions on Dielectrics and Electrical Insulation, vol. 12, no. 5, pp. 891-903, 2005.

[2] T. Liu, "Development and application of electrical equipment intelligent detection management system," in Proceedings of the 5th International Conference on Smart Grid and Electrical Automation (ICSGEA), pp. 70-73, June 2020.

[3] X. Tian, Z. Lan, F. Huo et al., "Calculation of electric field on substation equipment considering AC ion flow field," Mathematical Problems in Engineering, vol. 2020, Article ID 3914872, 2020.

[4] C. Shi, J. Fei, X. Zhang, Q. Yao, and J. Fan, "Continuous trust evaluation of power equipment and users based on risk measurement," Scientific Programming, vol. 2020, Article ID 8895804, 2020.

[5] X. Wang, Y. Chen, W. Ruan, Q. Gao, G. Ying, and Li Dong, "Intelligent detection and recovery of missing electric load data based on cascaded convolutional autoencoders," Scientific Programming, vol. 2020, Article ID 8828745, 2020.

[6] C. Guo, M. Ren, C. Xia, M. Dong, and B. Wang, "Fault diagnosis of power equipment based on infrared image analysis," in Proceedings of the IEEE International Conference on Advances in Electrical Engineering and Computer Applications (AEECA), pp. 659-663, Dalian, Chinapp, Aug 2020.

[7] N. K. Dhote and J. B. Helonde, "Fuzzy algorithm for power transformer diagnostics," Advances in Fuzzy Systems, vol. 2013, Article ID 421621, 7 pages, 2013.

[8] Q. Wang, S. Wang, R. Shi, and Y. Li, "A power transformer fault diagnosis method based on random vector functionallink neural network," Mathematical Problems in Engineering, vol. 2021, Article ID 6656061, 2021.

[9] K. Liu, S. Wu, Z. Luo et al., "An intelligent fault diagnosis method for transformer based on ipso-gcforest," Mathematical Problems in Engineering, vol. 2021, Article ID 6610338, 2021.

[10] J. Yang, B. Liu, C. Liu, F. Lu, and W. Rong, "The application of evidence theory in the field of equipment fault diagnosis," in Proceedings of the 2006 6th World Congress on Intelligent Control and Automation, pp. 5824-5827, Dalian, China, June 2006.

[11] G. Wang, C. Feng, X. Mu, and H. Liu, "Intelligent diagnosis of power transformer working fault," in Proceedings of the 2019 Chinese Control And Decision Conference (CCDC), pp. 383387, Nanchang, China, June 2019.

[12] Y. Wang, F. Lu, and H. Li, "The fault diagnosis method for electrical equipment based on Bayesian network," in Proceedings of the2005 International Conference on Electrical Machines and Systems, pp. 2259-2261, September 2005.

[13] Y. Lin, W. Zhang, H. Zhang, D. Bai, J. Li, and R. Xu, "An intelligent infrared image fault diagnosis for electrical equipment," in Proceedings of the 2020 5th Asia Conference on 
Power and Electrical Engineering (ACPEE), pp. 1829-1833, Chengdu, China, June 2020.

[14] W. Yongqiang, L. Fangcheng, and L. Heming, "The fault diagnosis method for electrical equipment using bayesian network," in Proceedings of the2009 First International Workshop on Education Technology and Computer Science, pp. 563-565, Wuhan, China, March 2009.

[15] H. Zou and F. Huang, "A novel intelligent fault diagnosis method for electrical equipment using infrared thermography," Infrared Physics \& Technology, vol. 73, pp. 29-35, 2015.

[16] L. Yifei, Z. Hangyan, and Y. Yujie, "Fault diagnosis algorithm of Electrical system based on Deep Learning," in Proceedings of the2020 2nd International Conference on Information Technology and Computer Application (ITCA), pp. 226-228, Guangzhou, China, December 2020.

[17] A. Jiang, N. Yan, B. Shen, C. Gu, H. Zhu, and H. Huang, "Research on infrared image recognition method of power equipment based on deep learning," in Proceedings of the 2020 IEEE International Conference on High Voltage Engineering and Application (ICHVE), pp. 1-4, September 2020.

[18] X. Gong, Q. Yao, M. Wang, and Y. Lin, "A deep learning approach for oriented electrical equipment detection in thermal images," IEEE Access, vol. 6, pp. 41590-41597, 2018.

[19] C. Wei, F. Tao, Y. Lin et al., "Substation equipment thermal fault diagnosis model based on ResNet and improved bayesian optimization," in Proceedings of the 2019 9th International Conference on Power and Energy Systems (ICPES), pp. 1-5, Perth, December 2019.

[20] S.M. Suh, Y. Park, K.M. Ko et al., "Weighted mask R-CNN for improving adjacent boundary segmentation," Journal of Sensors, vol. 2021, Article ID 8872947, 8 pages, 2021.

[21] S. Ren, K. He, R. Girshick, and J. Sun, "Faster R-CNN: towards real-time object detection with region proposal networks," IEEE Transactions on Pattern Analysis and Machine Intelligence, vol. 39, no. 6, pp. 1137-1149, 2017.

[22] Y. Cheng, L. Xia, B. Yan, J. Chen, D. Hu, and L. Zhu, “A defect detection method based on faster RCNN for power equipment," Journal of Physics: Conf. Ser, vol. 1754, Article ID 012025, 2021.

[23] X. Xu, L. Yang, and F. Yang, "Railway subgrade defect automatic recognition method based on improved faster R-CNN," Scientific Programming, vol. 2018, Article ID 4832972, 2018.

[24] J. Wright, A. Y. Yang, A. Ganesh, S. S. Sastry, and Y. Ma, "Robust face recognition via sparse representation," IEEE Transactions on Pattern Analysis and Machine Intelligence, vol. 31, no. 2, pp. 210-227, 2009.

[25] J. J. Thiagarajan, K. N. Ramamurthy, P. Knee, A. Spanias, and V. Berisha, "Sparse representations for automatic target classification in SAR images," in Proceedings of the 4th Int. Symp. Commun., Control Signal Process., pp. 1-4, Limassol, Cyprus, March 2010.

[26] L. Zhang, Z. Tao, and B. Wang, "SAR image target recognition using kernel sparse representation based on reconstruction coefficient energy maximization rule," in Proceedings of the 2016 IEEE International Conference on Acoustics, Speech and Signal Processing (ICASSP), pp. 2369-2373, Shanghai, China, March 2016.

[27] X. Xing, K. Ji, H. Zou, and J. Sun, "Sparse representation based SAR vehicle recognition along with aspect angle," The Scientific World Journal, vol. 2014, Article ID 834140, 2014. 